TAIWANESE JOURNAL OF MATHEMATICS

Vol. 16, No. 3, pp. 885-900, June 2012

This paper is available online at http://journal.taiwanmathsoc.org.tw

\title{
MULTIPLE SOLUTIONS OF THE STEADY FLOWS IN A RECTANGULAR CHANNEL WITH SLIP EFFECT ON TWO EQUALLY POROUS WALLS
}

\author{
Un-Un Kuo and Ching-An Wang
}

\begin{abstract}
We study the boundary layer equation $f^{\prime \prime \prime}(\eta)+R\left(\left(f^{\prime}(\eta)\right)^{2}-f(\eta) f^{\prime \prime}(\eta)\right)$ $=K$, subjects to the boundary conditions $f(0)=f^{\prime \prime}(0)=0, f(1)=1$ and $f^{\prime}(1)+\varphi f^{\prime \prime}(1)=0$. The given problem arises from the study of steady laminar flows in channels with two equally porous walls, where $R$ relates to the Reynold's number, and $K$ is an integration constant. We are able to obtain the homogeneity property and classify all types of solutions for the prescribed positive slip coefficient $\varphi$. In particular, the existence of the continuums in the $R-K$ plane has been verified, and this leads to the existence of multiple solutions for large $R$.
\end{abstract}

\section{INTRODUCTION}

The Navier-Stokes equation describes the two-dimensional flows in a rectangular channel with porous walls. By applying a similarity transformation [1], the governing equation for steady, incompressible, axis-symmetric laminar flow in a channel with two porous walls could be reduced to

$$
f^{\prime \prime \prime}(\eta)+R\left(\left(f^{\prime}(\eta)\right)^{2}-f(\eta) f^{\prime \prime}(\eta)\right)=K .
$$

Here $K$ is an integration constant and $R$ corresponds to the cross flow Reynold number based on wall velocity (filtration Reynold number), while positive (negative) $R$ represents the suction (injection) through the walls. The function $f$ is related to the stream function, $\eta$ is the normalized transverse coordinate, namely, $\eta= \pm 1$ at the wall.

If the flow is assumed to be antisymmetric, then $f=f^{\prime \prime}=0$ should be imposed at the central line $\eta=0$. Therefore, the steady flows could be studied from the boundary value problem (BVP) of (1.1), subjects to the boundary conditions:

Received October 7, 2010, accepted February 21, 2011.

Communicated by Jong-Shenq Guo.

2010 Mathematics Subject Classification: 34B15, 76R10, 76R50.

Key words and phrases: Shooting method, Classification, Homogeneity.

The author for correspondence, and this work is supported in part by National Sciences Council, Taiwan, under the project NSC-98-2115-M194-008. 


$$
f(0)=f^{\prime \prime}(0)=0, \quad f(1)=1, \quad f^{\prime}(1)+\varphi f^{\prime \prime}(1)=0,
$$

where positive $\varphi$ is the slip coefficient.

It is clear that $f(\eta)=\left[K \eta^{3}+(6-K) \eta\right] / 6$, with $K=-3 /(1+3 \varphi)$, is the solution of (BVP) when $R=0$. For nonzero $R$, as in $[2,4]$, suppose we set $f(\eta)=(b / R) g(\xi)$, $\xi=b \eta$ for some positive $b$ which is to be determined. Then, $g(\xi)$ satisfies the following associated boundary value problem $\left(\mathrm{BVP}_{1}\right)$ :

$$
\begin{gathered}
g^{\prime \prime \prime}(\xi)+\left(g^{\prime}(\xi)\right)^{2}-g(\xi) g^{\prime \prime}(\xi)=R K / b^{4} \equiv \beta, \\
g(0)=g^{\prime \prime}(0)=0, \\
g(b)=R / b, \quad g^{\prime}(b)+\varphi b g^{\prime \prime}(b)=0 .
\end{gathered}
$$

By assigning values to $\alpha, \beta$ with

$$
g^{\prime}(0)=\alpha, \quad g^{\prime \prime \prime}(0)=\beta-\alpha^{2},
$$

one can integrate (1.3), with the initial values (1.4) and (1.6). Let $g(\xi ; \alpha, \beta)$ be the solution of the initial value problem (1.3), (1.4) and (1.6). Suppose, given a prescribed positive $\varphi, g^{\prime}(b ; \alpha, \beta)+\varphi b g^{\prime \prime}(b ; \alpha, \beta)=0$ holds at $\xi=b^{*}$, then (BVP) will possess a solution with $R=b^{*} g\left(b^{*} ; \alpha, \beta\right)$ and $K=\left(b^{*}\right)^{4} \beta / R$.

Note that $g\left(\xi ; \alpha, \alpha^{2}\right)=\alpha \xi$ is not a solution of $\left(\mathrm{BVP}_{1}\right)$. Therefore, we will classify the type of solutions of $\left(\mathrm{BVP}_{1}\right)$ by assigning $\alpha, \beta$ from the following sets:

$$
\begin{aligned}
& D_{1}=\left\{(\alpha, \beta) \in \mathbb{R}^{2}: \alpha \leq 0, \beta<\alpha^{2}\right\}, \\
& D_{2}=\left\{(\alpha, \beta) \in \mathbb{R}^{2}: \alpha>0, \beta<\alpha^{2}\right\}, \\
& D_{3}=\left\{(\alpha, \beta) \in \mathbb{R}^{2}: \alpha \geq 0, \beta>\alpha^{2}\right\}, \\
& D_{4}=\left\{(\alpha, \beta) \in \mathbb{R}^{2}: \alpha<0, \beta>\alpha^{2}\right\} .
\end{aligned}
$$

Moreover, based on the homogeneity of $g(\xi ; \alpha, \beta)$, as in [4], the following main result will be verified:

\section{Main Result:}

There exist two continuums $\Gamma_{*}$ and $\Gamma^{*}$ in the $R-K$ plane such that (BVP) has a solution if and only if the pair $(R, K)$ lies on $\Gamma_{*} \cup \Gamma^{*}$. For each $(R, K)$ lying on the branch $\Gamma_{*},(\mathrm{BVP})$ possesses non-negative and concave solutions, while non-negative and non-concave solution or non-monotone solutions exist when $(R, K)$ lies on $\Gamma^{*}$. In particular, when the slip effect is low, (BVP) possesses at least three suctive solutions of different type for sufficiently large $R$. 


\section{Classification}

\subsection{Preliminary results}

For the convenience, we denote the problem of (1.3), (1.4) and (1.6) by (IVP). Note that the existence of solution $g\left(b^{*} ; \alpha, \beta\right)$ of (IVP) is independent of the choice of $\varphi$. Therefore, we recall some important properties of $g\left(b^{*} ; \alpha, \beta\right)$ from the study of $\left(\mathrm{BVP}_{1}\right)$ with $\varphi=0$ in [2].

Proposition 2.1. ([2]). Suppose $\beta \neq \alpha^{2}$. Then, the following properties hold:

(a) $g^{(i v)}(\xi)<0$ for all positive $\xi$;

(b) $g^{\prime}(\xi)$ has no positive zero for $(\alpha, \beta) \in D_{1}$;

(c) $g^{\prime}(\xi)$ has exactly one positive zero for $(\alpha, \beta) \in D_{2}$;

(d) $g^{(k)}(\xi)$ has exactly one positive zero $d_{k}, k=1,2,3$, respectively, with $d_{3}<$ $d_{2}<d_{1}$, for $(\alpha, \beta) \in D_{3}$;

(e) $g^{\prime}(\xi)$ has exactly two positive zeros, $0<a_{1}<a_{2}$, and $g^{(k)}(\xi)$ has exactly one positive zero $d_{k}^{*}$ for $k=2,3$, if $(\alpha, \beta) \in D_{4}$. Furthermore, $a_{1}<d_{3}^{*}<d_{2}^{*}<a_{2}$.

From Proposition 2.1, the selected graphs of $g(\xi ; \alpha, \beta)$ for some $(\alpha, \beta) \in D_{i}$ 's are shown in Figure 2.1, respectively. Let $\psi(\xi):=\psi(\xi ; \varphi)=g^{\prime}(\xi)+\varphi \xi g^{\prime \prime}(\xi)$ and $c(\alpha, \beta):=c(\alpha, \beta ; \varphi)$ be a positive zero of $\psi(\xi)$ for a prescribed positive $\varphi$. We are in position to explore the existence of zeros of $\psi(\xi)=0$ and classify the types of solutions for (BVP) in the next section.

\subsection{The roots of $\psi(\xi)=0$}

By choosing $(\alpha, \beta)$ from $D_{i}, i=1, \cdots, 4$, the existence of roots of $\psi(\xi)=0$ will be discussed in the following lemmas.

Lemma 2.1. If $(\alpha, \beta) \in D_{1}$, then $\psi(\xi)$ has no positive zero.

Proof. It is clear that $g(\xi), g^{\prime}(\xi), g^{\prime \prime}(\xi), g^{\prime \prime \prime}(\xi)$ are negative initially, since $g^{\prime}(0)=\alpha \leq 0$ and $g^{\prime \prime \prime}(0)=\beta-\alpha^{2}<0$. This implies that $\psi(0)=\alpha \leq 0$ and $\psi(\xi)=g^{\prime}(\xi)+\varphi \xi g^{\prime \prime}(\xi), \psi^{\prime}(\xi)=g^{\prime \prime}(\xi)(1+\varphi)+\varphi \xi$ are also negative initially. In fact, from Proposition 2.1(a), (b), it is clear that $g, g^{\prime}, g^{\prime \prime}$ and $g^{\prime \prime \prime}$ are negative for all $\xi>0$. This implies that $\psi(\xi)$ is strictly decreasing for $\xi>0$. Thus, for any prescribed positive $\varphi, \psi(\xi)$ has no positive zero.

Lemma 2.2. If $(\alpha, \beta) \in D_{2}$, then $\psi(\xi)$ has exactly one positive zero. 


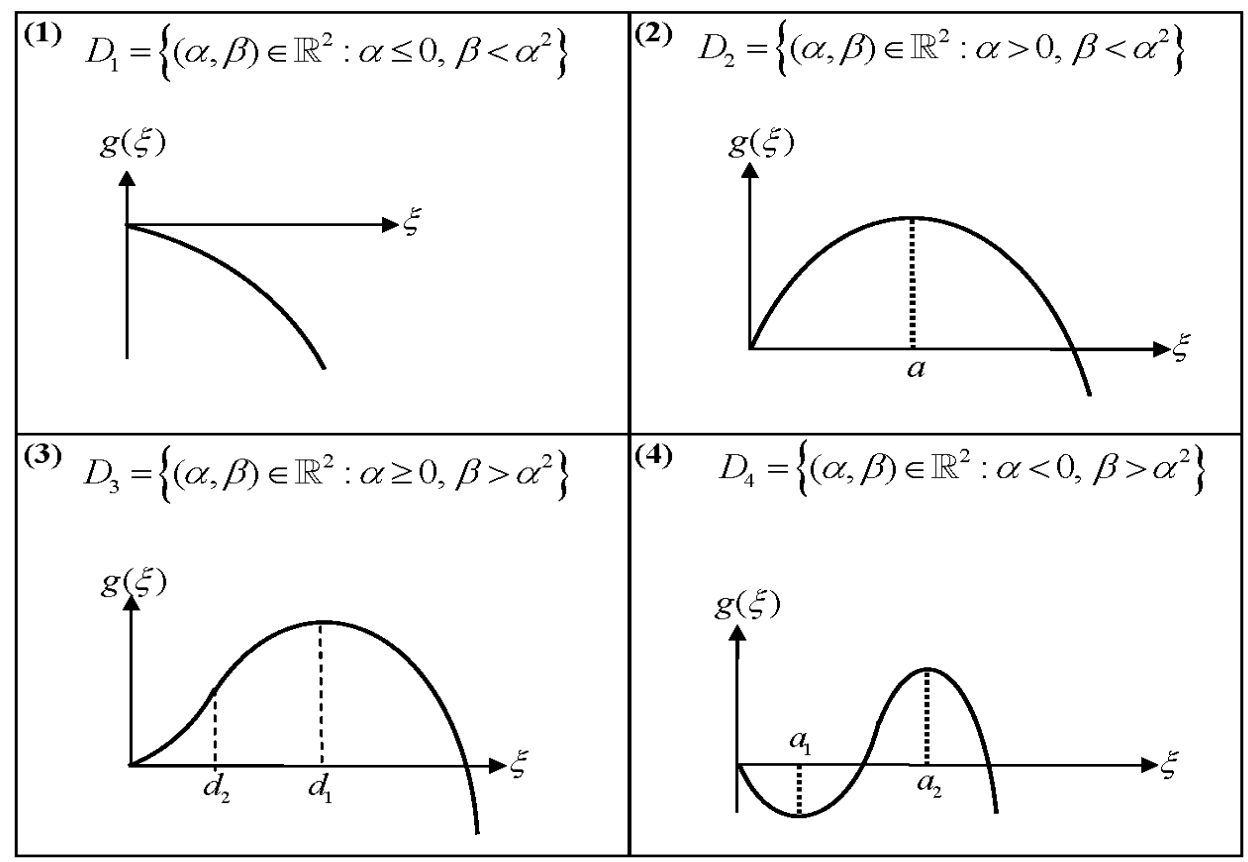

Fig. 2.1. The selected graphs of $g(\xi ; \alpha, \beta)$ for some $(\alpha, \beta) \in D_{i}^{\prime} s$

Proof. It is clear that $g(\xi), g^{\prime}(\xi)$ are positive, and $g^{\prime \prime}(\xi), g^{\prime \prime \prime}(\xi)$ are negative initially, since $g^{\prime}(0)=\alpha>0$ and $g^{\prime \prime \prime}(0)=\beta-\alpha^{2}<0$. This implies that $\psi(0)=$ $\alpha>0$ and $\psi^{\prime}(\xi)$ is negative initially. In fact, from Proposition 2.1(a), (c), it is clear that $g^{\prime \prime}(\xi)<0, g^{\prime \prime \prime}(\xi)<0$ for $\xi>0$, and this implies that $\psi^{\prime}(\xi)<0$ and $\psi(\xi)^{\prime \prime}=g^{\prime \prime \prime}(\xi)(1+2 \varphi)+\varphi \xi g^{(i v)}(\xi)<0$ for $\xi>0$. This implies that $\psi(\xi)$ is strictly decreasing and concave for $\xi>0$. Hence, for the prescribed positive $\varphi, \psi(\xi)$ has exactly one positive zero $c(\alpha, \beta)$. In particular, $c(\alpha, \beta)<d_{1}$ since $d_{1}$ is the unique zero of $g^{\prime}(\xi)$ and $\psi\left(d_{1}\right)<0$.

Remark 2.1. From Proposition 2.1(c) and Lemma 2.2, we have $g(c(\alpha, \beta))>0$, and it yields that $R=c(\alpha, \beta) g(c ; \alpha, \beta)$ is positive. Furthermore, $K=c^{4}(\alpha, \beta) \beta / R$ is positive if $\beta>0$, and negative if $\beta<0$. In fact, from $f(\eta)=c(\alpha, \beta) g(\xi ; \alpha, \beta) / R$ with $\xi=c \eta$, the corresponding solution $f(\eta)$ of (BVP) is nonnegative and concave for $(\alpha, \beta) \in D_{2}$.

Lemma 2.3. If $(\alpha, \beta) \in D_{3}$, then $\psi(\xi)$ has exactly one positive zero.

Proof. It is clear that $g, g^{\prime}, g^{\prime \prime}, g^{\prime \prime \prime}$ are positive on initially, since $g^{\prime}(0)=\alpha \geq 0$ and $g^{\prime \prime \prime}(0)=\beta-\alpha^{2}>0$. This yields that $\psi(0)=\alpha \geq 0$, and $\psi, \psi^{\prime}, \psi^{\prime \prime}$ are also positive initially. Again, from Proposition 2.1(d), $g^{(k)}$ has exactly one positive zero $d_{k}$, 
$k=1,2,3$, with $d_{3}<d_{2}<d_{1}$. This implies that $\psi(\xi)>0$ on $\left(0, d_{2}\right)$, since on which $g$ and $g^{\prime}$ are positive. Also, from the facts that $g^{(k)}<0, k=2,3,4$, on $\left(d_{2}, \infty\right)$, we get that $\psi^{\prime}<0$, and $\psi^{\prime \prime}<0$ for $\xi>d_{2}$. This implies that $\psi(\xi)$ is strictly decreasing and concave on $\left(d_{2}, \infty\right)$. It is clear that $\psi\left(d_{2}\right)>0$ and $\psi\left(d_{1}\right)<0$. Hence, for any positive $\varphi, \psi(\xi ; \alpha, \beta)$ has the unique zero $c(\alpha, \beta)$ with $d_{2}<c(\alpha, \beta)<d_{1}$.

Remark 2.2. From Proposition 2.1(d) and Lemma 2.3, we have $g(c(\alpha, \beta) ; \alpha, \beta)>$ 0 , and it leads to the corresponding $R(\alpha, \beta)>0$ and $K(\alpha, \beta)>0$. That is, the corresponding suctive solution $f(\eta)$ of (BVP) is also nonnegative, non-concave on ( 0 , 1) for $(\alpha, \beta) \in D_{3}$.

Lemma 2.4. If $(\alpha, \beta) \in D_{4}$, then $\psi(\xi)$ has exactly two positive zeros.

Proof. It is clear that $g, g^{\prime}$ are negative and $g^{\prime \prime}, g^{\prime \prime \prime}$ are positive initially, since $g^{\prime}(0)=\alpha<0$ and $g^{\prime \prime \prime}(0)=\beta-\alpha^{2}>0$. This yields that $\psi(\xi)$ is negative initially, since $\psi(0)=\alpha<0$ although $\psi^{\prime}(\xi)>0$ initially. In fact, $\psi^{\prime}(\xi)>0$ on $\left(0, a_{1}\right)$ and $\psi\left(a_{1}\right)>0$. This implies that there is the first zero $c_{1}(\alpha, \beta)$, which is lying on $\left(0, a_{1}\right)$.

Now, on $\left(a_{1}, d_{3}^{*}\right)$, we have $\psi^{\prime}(\xi)>0$ since $g^{\prime \prime}, g^{\prime \prime \prime}$ are positive. This implies $\psi(\xi)>0$ on $\left(a_{1}, d_{3}^{*}\right)$. Also, on $\left(d_{3}^{*}, d_{2}^{*}\right)$, we get $\psi(\xi)>0$ since on which $g^{\prime}, g^{\prime \prime}$ are positive.

Moreover, we have $\psi\left(d_{2}^{*}\right)>0, \psi\left(a_{2}\right)<0$, and this yields the second zero $c_{2}(\alpha, \beta)$ of $\psi(\xi)$ which is lying on $\left(d_{2}^{*}, a_{2}\right)$. Also, it is clear that $\psi(\xi)$ is strictly decreasing and concave for $\xi>a_{2}$. Hence, $\psi(\xi)$ has exactly two zeros $c_{1}$, $c_{2}$ satisfying $0<c_{1}<a_{1}$, $d_{2}^{*}<c_{2}<a_{2}$, respectively.

Remark 2.3. It is clear that the corresponding pair $(R, K)$ from $c_{1}(\alpha, \beta)$ satisfies $R<0, K<0$ for $(\alpha, \beta) \in D_{4}$. Also, from $f(\eta)=c_{1}(\alpha, \beta) g(\xi ; \alpha, \beta) / R$ with $\xi=c_{1} \eta$ and $0<c_{1}<a_{1}$, this implies that the corresponding injective solution of (BVP) is nonnegative, and concave for $(\alpha, \beta) \in D_{4}$. Moreover, from (1.3), it is clear to obtain $g^{(i v)}\left(c_{2}\right)=g\left(c_{2}\right) g^{\prime \prime \prime}\left(c_{2}\right)+\varphi c_{2}\left(g^{\prime \prime}\left(c_{2}\right)\right)^{2}$. This implies that $g\left(c_{2}\right)>0$ since both $g^{(i v)}\left(c_{2}\right), g^{\prime \prime \prime}\left(c_{2}\right)$ are negative. Therefore, the existence of $c_{2}$ 's will lead to the non-monotone suctive solutions $f(\eta)$ of (BVP) with $R>0, K>0$.

Note that the distribution of $c(\alpha, \beta)$ 's, the zeros of $g^{(k)}(\xi), k=1,2,3$, and the corresponding profiles of $f(\eta)$ are shown in Figure 2.2. Therefore, we conclude that (BVP) can only possess the following types of solutions:

(I) $f$ is a nonnegative and concave ;

(II) $f$ is nonnegative and non-concave, on which there exists an $\eta_{1} \in(0,1)$ such that $f^{\prime \prime}>0$ on $\left(0, \eta_{1}\right)$ and $f^{\prime \prime}<0$ on $\left(\eta_{1}, 1\right)$;

(III) $f$ is non-monotone on which

(i) there exist an $\eta_{1} \in(0,1)$ such that $f^{\prime \prime}>0$ on $\left(0, \eta_{1}\right)$ and $f^{\prime \prime}<0$ on $\left(\eta_{1}, 1\right)$;

(ii) there exist an $\eta_{2} \in\left(0, \eta_{1}\right)$ such that $f<0$ on $\left(0, \eta_{2}\right)$ and $f>0$ on $\left(\eta_{2}, 1\right)$. 
Furthermore, also from Remarks 2.1 and 2.3, (BVP) possesses no nonnegative and concave solution for any pair $(R, K)$ when $R<0$ and $K>0$.
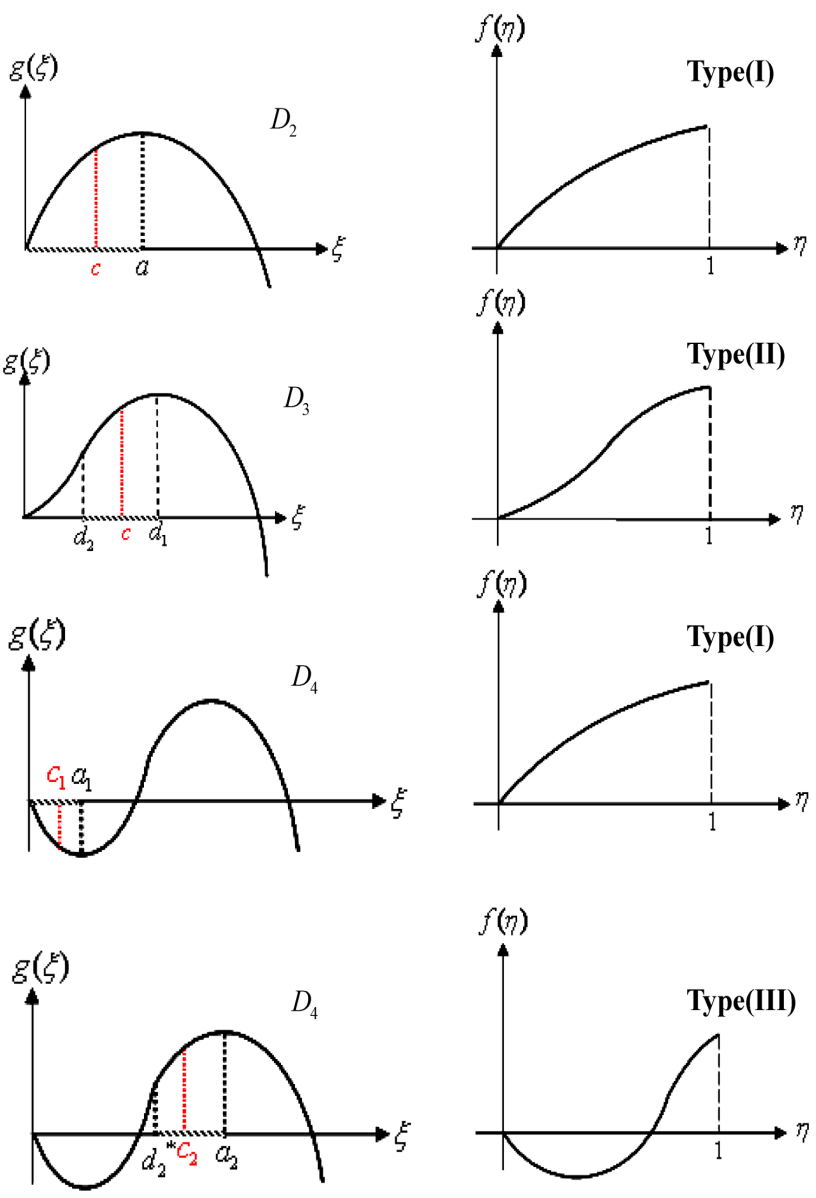

Fig. 2.2. The distribution of $c(\alpha, \beta)$ and the profile of the corresponding solutions of (BVP).

\section{SOlutions OF (BVP)}

As mentioned earlier, $\psi(\xi)$ possesses unique positive zero $c(\alpha, \beta)$ for $(\alpha, \beta) \in D_{2}$, $D_{3}$, or two positive zeros $c_{1}(\alpha, \beta)<c_{2}(\alpha, \beta)$ for $(\alpha, \beta) \in D_{4}$. All the zeros of $\psi(\xi)$ will lead to the solutions of (BVP) with the corresponding $R(\alpha, \beta), K(\alpha, \beta)$. For the convenience, define the solution sets for (BVP) in the $R-K$ plane by

$$
\begin{array}{ll}
\Gamma_{1}=\left\{\vec{x}(\alpha, \beta):(\alpha, \beta) \in D_{2}\right\}, & \Gamma_{2}=\left\{\overrightarrow{x_{1}}(\alpha, \beta):(\alpha, \beta) \in D_{4}\right\}, \\
\Gamma_{3}=\left\{\vec{x}(\alpha, \beta):(\alpha, \beta) \in D_{3}\right\}, & \Gamma_{4}=\left\{\overrightarrow{x_{2}}(\alpha, \beta):(\alpha, \beta) \in D_{4}\right\},
\end{array}
$$


where $\vec{x}(\alpha, \beta)=(R(\alpha, \beta), K(\alpha, \beta))$ for $(\alpha, \beta) \in D_{2} \cup D_{3}$ and $\overrightarrow{x_{i}}(\alpha, \beta)=\left(R_{i}(\alpha, \beta)\right.$, $\left.K_{i}(\alpha, \beta)\right), i=1,2$, corresponding to $c_{1}(\alpha, \beta)<c_{2}(\alpha, \beta)$ for $(\alpha, \beta) \in D_{4}$. In order to verify that $\Gamma_{k}$ 's are connected, it is required to achieve the homogeneity property in the next section.

\subsection{Homogeneity}

As in [2], [4], let $h(\xi)=g(\xi / \lambda ; \alpha, \beta) / \lambda$, and $\lambda$ be a positive constant. Then, $h(\xi)$ satisfies the equation

$$
h^{\prime \prime \prime}(\xi)+\left(h^{\prime}(\xi)\right)^{2}-h(\xi) h^{\prime \prime}(\xi)=\beta / \lambda^{4},
$$

subjects to $h(0)=0, h^{\prime}(0)=\alpha / \lambda^{2}, h^{\prime \prime}(0)=0$ and $h^{\prime \prime \prime}(0)=\left(\beta-\alpha^{2}\right) / \lambda^{4}$. This gives the homogeneity property of $g(\xi)$ as described below.

Lemma 3.5. For all $\lambda>0, g(\xi ; \alpha, \beta)=\lambda g\left(\lambda \xi ; \alpha / \lambda^{2}, \beta / \lambda^{4}\right)$.

Also, let $a(\alpha, \beta)$ be a positive zero of $g^{\prime}(\xi ; \alpha, \beta)$, if it does exist. Therefore, we have the following homogeneity properties for $c(\alpha, \beta), a(\alpha, \beta), R(\alpha, \beta)$ and $K(\alpha, \beta)$ by

$$
\begin{gathered}
c(\alpha, \beta)=c\left(\alpha / \lambda^{2}, \beta / \lambda^{4}\right) / \lambda, \\
a(\alpha, \beta)=a\left(\alpha / \lambda^{2}, \beta / \lambda^{4}\right) / \lambda, \\
R(\alpha, \beta)=R\left(\alpha / \lambda^{2}, \beta / \lambda^{4}\right), \\
K(\alpha, \beta)=K\left(\alpha / \lambda^{2}, \beta / \lambda^{4}\right),
\end{gathered}
$$

for all $\lambda>0$ and $(\alpha, \beta) \in D_{i}, i=2,3,4$.

It should be pointed out that the obtained homogeneity yields that the corresponding $(R(\alpha, \beta), K(\alpha, \beta))$ 's are the same for any $(\alpha, \beta)$ lying on a given parabola $\beta=\gamma \alpha^{2}$ for some $\gamma \neq 1$ in $D_{2}, D_{3}$ or $D_{4}$. Therefore, $\Gamma_{k}$ 's can be rewritten as

$$
\begin{gathered}
\Gamma_{1}=\{\vec{x}(1, \gamma):-\infty<\gamma<1\}, \quad \Gamma_{2}=\left\{\overrightarrow{x_{1}}(\gamma, 1): \gamma \in(-1,0)\right\}, \\
\Gamma_{3}=\{\vec{x}(\gamma, 1): \gamma \in[0,1)\}, \quad \Gamma_{4}=\left\{\overrightarrow{x_{1}}(\gamma, 1): \gamma \in(-1,0)\right\} .
\end{gathered}
$$

Moreover, the homogeneity also yields an efficient numerical strategy in obtaining the graphs of $\Gamma_{k}$ 's. That is, instead of choosing $(\alpha, \beta)$ randomly, one may choose them along some simple curves on $D_{i}$ 's. Consequently, as shown in Figures 3.1, 3.2, the bifurcation diagrams of (BVP) do exhibit the existence of continuums of $(R, K)$, and it will be verified in the following sections. 


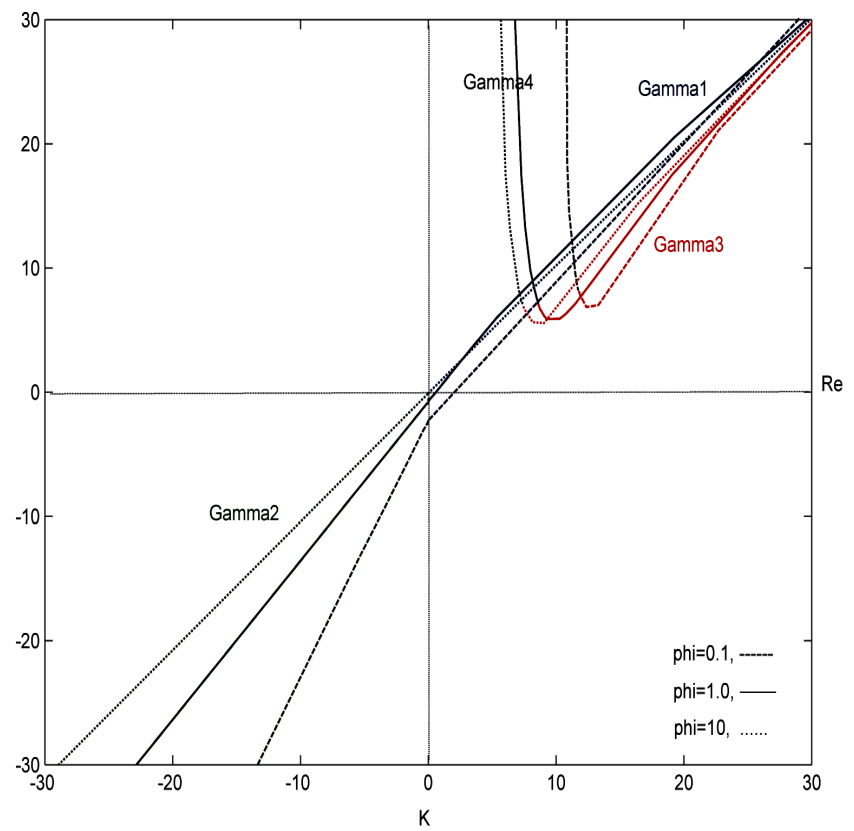

Fig. 3.1. The bifurcation diagram of (BVP) for various positive $\varphi$ 's.

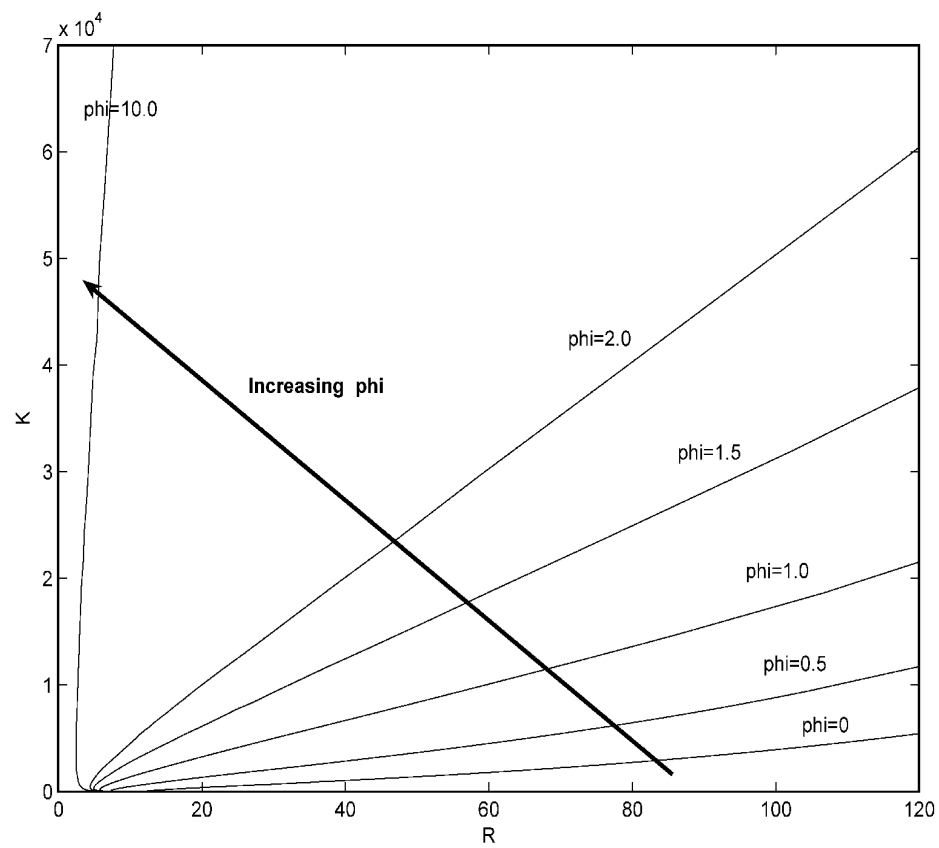

Fig. 3.2. The detail diagram of the branch $\Gamma_{4}$ of (BVP) for various $\varphi$ 's. 


\subsection{Nonnegative and concave solutions}

It is clear that, from Remark 2.1, the corresponding $(R, K)$ 's for $(\alpha, \beta) \in D_{2}$ will lead to the type (I) solutions of (BVP). Therefore, we first have the following theorem for the suctive solutions.

Theorem 3.1. (BVP). Possesses a nonnegative and concave solution with suction if and only if $(R, K) \in \Gamma_{1}$.

Proof. We omit the proof of the sufficient condition, since it can be obtained directly from Lemma 2.2 and Remark 2.1.

To verify the necessary condition, let $f(\eta)$ be a type (I) solution of (BVP) with $R>0$. It is clear that $f^{\prime}(0)$ is positive initially. Suppose $f^{\prime}(0)=\mu, b=\sqrt{\mu R}$ and $g(\xi)=R f(\xi / b) / b$, we get $g^{\prime}(\xi)=f^{\prime}(\xi / b) / \mu$, and it yields $\alpha=g^{\prime}(0)=f^{\prime}(0) / \mu=1$. Now, we need only to prove that $\beta<1$. To see this, from the facts that $f^{\prime \prime}(0)=0$, $f^{\prime}(0)=\mu>0$ and $f(\eta)$ is a concave solution of (BVP), it yields $f^{\prime \prime \prime}(0)<0$. This implies that $f^{\prime \prime \prime}(0)=K-R \mu^{2}<0$ and $\beta=K /\left(R \mu^{2}\right)<1$.

To explore the connected property of $\Gamma_{1}$, the limiting behavior of $(R, K)$ as $\gamma$ tends to $1^{-}$and $-\infty$ should be discussed.

Corollary 3.1. (a). $\lim _{\gamma \rightarrow 1^{-}} c(1, \gamma)=+\infty ;$ (b). $\lim _{\gamma \rightarrow-\infty} c(1, \gamma)=0$.

Proof. To prove assertion (a), we recall that $g^{\prime}(\xi ; 1,1)=1$, and $g^{\prime \prime}(\xi ; 1,1)=0$. Then, by the continuous dependence on initial data, given any $\varepsilon>0$, there is a $\delta>0$ such that $\left|1-g^{\prime}(c(1, \gamma) ; 1, \gamma)\right|<\varepsilon$ and $\left|0-g^{\prime \prime}(c(1, \gamma) ; 1, \gamma)\right|<\varepsilon$, whenever $\gamma \in$ $(1-\delta, 1)$. This implies that, for $\gamma \in(1-\delta, 1), c(1, \gamma)=-g^{\prime}(c(1, \gamma)) / \varphi g^{\prime \prime}(c(1, \gamma)) \geq$ $-(1-1 / \varepsilon) / \varphi$ and $\lim _{\gamma \rightarrow 1^{-}} c(1, \gamma)=+\infty$ is obtained.

We turn to verify assertion (b). From (3.1), $c(1, \gamma) \sqrt[4]{|\gamma|}=c(1 / \sqrt{|\gamma|},-1)$, if $\gamma<0$. Instead of study the limit of $c(1, \gamma)$ directly as $\gamma$ tends to $-\infty$, one can analyze the limit of $c(1 / \sqrt{|\gamma|},-1)$ if it does exist. By Lemma 2.2 , we have $c(1 / \sqrt{|\gamma|},-1)<$ $a(1 / \sqrt{|\gamma|},-1)$, where $a$ is the zero of $g^{\prime}$. Then, we have $\lim _{\gamma \rightarrow-\infty} c(1 / \sqrt{|\gamma|},-1)=0$ since $\lim _{\gamma \rightarrow \infty} a(1 / \sqrt{|\gamma|},-1)=0$ holds from Corollary 3.2 in [4]. Hence, the desired limit is obtained.

Now the limits of $(R, K)$, as $\gamma$ tends to $1^{-}$and $-\infty$, can be obtained from next corollary.

Corollary 3.2. (a). $\lim _{\gamma \rightarrow 1^{-}} R(1, \gamma)=+\infty, \lim _{\gamma \rightarrow 1^{-}} K(1, \gamma)=+\infty$;

(b). $\lim _{\gamma \rightarrow-\infty} R(1, \gamma)=0, \lim _{\gamma \rightarrow-\infty} K(1, \gamma)=-3 /(1+3 \varphi)$. 
Proof. To prove assertion (a), we also recall the results of Proposition 2.1(a), (c) and Lemma 2.2 that $g^{\prime}(\xi ; 1, \gamma)$ is concave and decreasing on $(0, c(1, \gamma))$. This yields $c(1, \gamma) \geq g(c(1, \gamma) ; 1, \gamma) \geq c(1, \gamma) / 2$, and, consequently, $R=c(1, \gamma) g(c(1, \gamma) ; 1, \gamma) \geq$ $c^{2}(1, \gamma) / 2$ and $K=\beta c^{4}(1, \gamma) / R \geq \gamma c^{2}(1, \gamma)$. Hence, we get the desired limits of $R$, $K$, as $\gamma$ tends to $1^{-}$.

To verify assertion (b), from the fact that $0<g(c(1, \gamma) ; 1, \gamma) \leq c(1, \gamma)$, we get

$$
0<R=c(1, \gamma) g(c(1, \gamma) ; 1, \gamma) \leq c(1, \gamma)^{2},
$$

and, then, the desired limit of $R$ is obtained, since $c(1, \gamma)$ tends to 0 as $\gamma$ tending to $-\infty$.

To obtain the desired limit of $K$, by integrating (1.3) and applying the initial conditions (1.4), (1.6), we get

$$
\begin{gathered}
g^{\prime \prime}(c)=\beta c-\int_{0}^{c} G(\eta) d \eta, \\
g^{\prime}(c)=\frac{\beta c^{2}}{2}+\alpha-\int_{0}^{c} G(\eta)(c-\eta) d \eta, \\
g(c)=\frac{\beta c^{3}}{6}+\alpha c-\int_{0}^{c} G(\eta) \frac{(c-\eta)^{2}}{2} d \eta,
\end{gathered}
$$

where $\left.G(\eta)=\left(g^{\prime}(\eta)\right)^{2}-g(\eta) g^{\prime \prime}(\eta)\right)$. Then, from (3.5)-(3.7), we further have

$$
\int_{0}^{c} G(\eta)\left(c^{2} \varphi+\frac{c^{2}-\eta^{2}}{2}\right) d \eta=g(c)[1+K(3 \varphi+1) / 3] .
$$

Also, from Proposition 2.1(a), we have $G(0)=\alpha^{2}$ and $G(\eta)$ is increasing. This implies that $\alpha^{2} \leq G(\eta) \leq\left(g^{\prime}(c)^{2}-g(c) g^{\prime \prime}(c)\right)$ on $[0, c]$. Then, also from (3.8), we get that

$$
|1+K(3 \varphi+1) / 3| \leq \frac{c^{3}(\varphi+1 / 3)\left[g^{\prime}(c)^{2}-g(c) g^{\prime \prime}(c)\right]}{g(c)} .
$$

Note that (3.9) holds for $c=c(1, \gamma)$. Then, as $c$ tending to 0 , we get

$$
\left.G(\eta)=\left(g^{\prime}(\eta)\right)^{2}-g(\eta) g^{\prime \prime}(\eta)\right) \rightarrow 1, \frac{c}{g(c)} \rightarrow 1,
$$

and the right-hand side of (3.9) tends to 0 , as $\gamma$ tending to $-\infty$. Thus, the desired limit of $K$ is obtained.

Note that, from the continuity, it is clear that $\Gamma_{1}$ is a continuum of one parameter in the half plane of $R>0, K \in \mathbb{R}$ connecting limit points $(0,-3 /(1+3 \varphi))$ and $(\infty, \infty)$. Moreover, (BVP) possesses at least one type (I) suctive solution for any positive $R$.

As in Theorem 3.1, the existence of type (I) injective solutions is obtained from the next theorem. 
Theorem 3.2. (BVP). has a nonnegative and concave solution with injection if and only if $(R, K) \in \Gamma_{2}$.

Proof. As in Theorem 3.1, we omit the proof of the sufficient condition. To show the necessary condition, let $f(\eta)$ be a type (I) solution of (BVP). From the fact $R<0, K<0, \beta=1$ in (1.3) is trivial by setting $c=\sqrt[4]{R K}$. It remains to show $\alpha \in(-1,0)$. In fact, $f(\eta)$ is positive, concave initially, and therefore $f^{\prime}(0)=\mu>0$. From $f(\eta)=(c / R) g(\xi)$, we have $g^{\prime}(0)=\alpha=R \mu / c^{2}(\alpha, 1)<0$. Hence, $\alpha \in(-1,0)$ is obtained, since $g^{\prime \prime \prime}(0)=1-\alpha^{2}>0$.

To explore the connected property of $\Gamma_{2}$, the limiting behavior of $(R, K)$ as $\gamma$ tends to $-1^{+}$and $0^{-}$should be discussed.

Corollary 3.3. (a). $\lim _{\gamma \rightarrow-1^{+}} c_{1}(\gamma, 1)=+\infty ; \quad$ (b). $\lim _{\gamma \rightarrow 0^{-}} c_{1}(\gamma, 1)=0$.

Proof. To prove assertion (a), we recall that $g^{\prime}(\xi ;-1,1)=-1, g^{\prime \prime}(\xi ;-1,1)=0$. Now from the continuous dependence on initial data, it is clear that for any given $\varepsilon>0$, there is a $\delta>0$ such that $\left|g^{\prime}\left(c_{1}(\gamma, 1) ; \gamma, 1\right)-(-1)\right|<\varepsilon$ and $\mid g^{\prime \prime}(c(\gamma, 1) ; \gamma, 1)-$ $0 \mid<\varepsilon$ for $\gamma \in(-1,-1+\delta)$. This yields that, for $\gamma \in(-1,-1+\delta), c_{1}(\gamma, 1)=$ $-g^{\prime}\left(c_{1}(\gamma, 1)\right) /\left[\varphi g^{\prime \prime}\left(c_{1}(\gamma, 1)\right)\right] \geq(1-\varepsilon) /(\varphi \varepsilon)$, and, hence, $\lim _{\gamma \rightarrow-1^{+}} c_{1}(\gamma, 1)=+\infty$.

To verify assertion (b), we recall again, from Proposition $2.1(\mathrm{~d})$, that $d_{2}(0,1)<$ $d_{1}(0,1)$ where $d_{2}(0,1), d_{1}(0,1)$ are the roots of $g^{\prime \prime}(\xi ; 0,1)$ and $g^{\prime}(\xi ; 0,1)$ respectively. From $g^{\prime}(\varepsilon ; 0,1)>0$ for each $\varepsilon \in\left(0, d_{2}(0,1)\right)$ and the continuous dependence on initial data, we have that $g^{\prime}(\varepsilon ; \gamma, 1)>0$ when $\gamma$ is close to $0^{-}$. However, $g^{\prime}(\xi ; \gamma, 1)<0$ initially. This implies that $a_{1}(\gamma, 1) \in(0, \varepsilon)$ and $\lim _{\gamma \rightarrow 0^{-}} a_{1}(\gamma, 1)=0$. Thus, the desired limit is obtained since $c_{1}(\gamma, 1) \in\left(0, a_{1}(\gamma, 1)\right)$.

Now the limits of $R, K$ can be obtained easily by following the lines in Corollary 3.2 .

Corollary 3.4. (a). $\lim _{\gamma \rightarrow-1^{+}} R_{1}(\gamma, 1)=-\infty, \lim _{\gamma \rightarrow-1^{+}} K_{1}(\gamma, 1)=-\infty$; (b). $\lim _{\gamma \rightarrow 0^{-}} R_{1}(\gamma, 1)=0, \lim _{\gamma \rightarrow 0^{-}} K_{1}(\gamma, 1)=-3 /(1+3 \varphi)$.

Note that $\Gamma_{2}$ is a continuum in the quadrant of $R<0, K<0$, connecting two limit points $(-\infty,-\infty)$ and $(0,-3 /(1+3 \varphi))$. Set $\Gamma_{*}=\Gamma_{1} \cup(0,-3 / 1+3 \varphi) \cup \Gamma_{2}$. This shows that (BVP) has at least one nonnegative, concave solution for every real $R$. In fact, our result of $\Gamma_{*}$ is consistent with the graph given in Figure 3.3.

\subsection{Non-negative and non-concave solutions}

By following the lines of Theorem 3.2, the existence of type (II) solutions can be obtained from the next theorem.

Theorem 3.3. (BVP) possesses a nonnegative and non-concave solution with suction if and only if $(R, K) \in \Gamma_{3}$. 
Now the limits of $R, K$ can be obtained from the following corollaries.

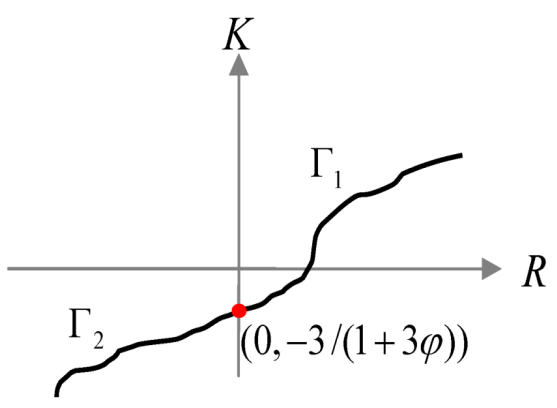

Fig. 3.3. $\Gamma_{*}$ is a continuum in the $R-K$ plane connecting the limit points $(-\infty,-\infty)$, $(+\infty,+\infty)$.

Corollary 3.5. (a). $\lim _{\gamma \rightarrow 1^{-}} c(\gamma, 1)=+\infty$;

(b). $\lim _{\gamma \rightarrow 0^{+}} c(\gamma, 1)=c(0,1)$, for some positive $c(0,1)$ varying in $\varphi$.

Proof. It is clear that $g^{\prime}(\xi ; 1,1) \equiv 1$ and $g^{\prime}(\xi ; 1,1) \equiv 0$ for all $\xi \geq 0$. By the continuous dependence, for $\varepsilon>0$, there is a $\delta>0$ such that $\left|1-g^{\prime}(c(\gamma, 1) ; \gamma, 1)\right|<\varepsilon$ and $\left|0-g^{\prime \prime}(c(\gamma, 1) ; \gamma, 1)\right|<\varepsilon$ for $\gamma \in(1-\delta, 1)$, where $c(\gamma, 1) \in\left(d_{2}(\gamma, 1), d_{1}(\gamma, 1)\right)$ and $d_{i}(\gamma, 1)$ 's are defined in Proposition 2.1(d). This implies $c(\gamma, 1)=-g^{\prime}(c(\gamma, 1)) / \varphi g^{\prime \prime}$ $(c(\gamma, 1)) \geq(1-\varepsilon) / \varphi \varepsilon$. Since $\varepsilon$ is arbitrarily small, thus $\lim _{\gamma \rightarrow 1^{-}} c(\gamma, 1)=+\infty$. Moreover, the assertion (b) is the direct consequence of the continuous dependence of $c(\alpha, \beta)$.

Corollary 3.6. (a). $\lim _{\gamma \rightarrow 1^{-}} R(\gamma, 1)=+\infty, \lim _{\gamma \rightarrow 1^{-}} K(\gamma, 1)=+\infty$;

(b). $\lim _{\gamma \rightarrow 0^{+}} R(\gamma, 1)=R(0,1), \lim _{\gamma \rightarrow 0^{+}} K(\gamma, 1)=K(0,1)$, for some positive $R(0,1), K(0,1)$ varying in $\varphi$.

Proof. To verify assertion (a), we first recall that $g^{\prime}(\xi ; 1,1) \equiv 1$, for all $\xi \geq 0$. By continuous dependence on initial data, given any $\varepsilon>0$, there is a $\delta>0$ such that $|g(\xi ; 1,1)-g(\xi ; \gamma, 1)|<\varepsilon$, for $\gamma \in(1-\delta, 1)$ and $|g(c(\gamma, 1) ; 1,1)-g(c(\gamma, 1) ; \gamma, 1)|<\varepsilon$. Then, we get $|c(\gamma, 1)-g(c(\gamma, 1) ; \gamma, 1)|<\varepsilon$, for $\gamma \in(1-\delta, 1)$. Now, from the expression of $R$, we get $R(\gamma, 1)=c(\gamma, 1) g(c(\gamma, 1) ; \gamma, 1)>c^{2}(\gamma, 1)-\varepsilon c(\gamma, 1)$, for $\gamma \in(1-\delta, 1)$. This implies that $\lim _{\gamma \rightarrow 1^{-}} R(\gamma, 1) \geq \lim _{\gamma \rightarrow 1^{-}} c^{2}(\gamma, 1)=+\infty$.

The assertion of $\lim _{\gamma \rightarrow 1^{-}} K(\gamma, 1)=+\infty$ could be obtained by the continuous dependence. That is, we get that

$$
\begin{aligned}
g(c(\gamma, 1) ; \gamma, 1) & =\int_{0}^{c(\gamma, 1)} g^{\prime}(\xi) d \xi \\
& \leq g^{\prime}\left(d_{2}(\gamma, 1) ; \gamma, 1\right) c(\gamma, 1)
\end{aligned}
$$




$$
\begin{aligned}
& =c(\gamma, 1)\left[\int_{0}^{d_{2}(\gamma, 1)} g^{\prime \prime}(\xi) d \xi+\gamma\right] \\
& <c(\gamma, 1)\left[c(\gamma, 1) g^{\prime \prime}\left(d_{3}(\gamma, 1) ; \gamma, 1\right)+\gamma\right] \\
& <c(\gamma, 1)\left[c^{2}(\gamma, 1)\left(1-\gamma^{2}\right)+\gamma\right] \\
& =c^{3}(\gamma, 1)\left(1-\gamma^{2}\right)+c(\gamma, 1) \gamma .
\end{aligned}
$$

This implies that $1 / R>1 /\left[c^{4}(\gamma, 1)\left(1-\gamma^{2}\right)+c^{2}(\gamma, 1) \gamma\right]$, and, consequently,

$$
K=\frac{c^{4}(\gamma, 1)}{R}>\frac{c^{4}(\gamma, 1)}{c^{4}(\gamma, 1)\left(1-\gamma^{2}\right)+c^{2}(\gamma, 1) \gamma}=\frac{1}{\left(1-\gamma^{2}\right)+\left[\gamma / c^{2}(\gamma, 1)\right]}
$$

when $\gamma$ sufficiently close to $1^{-}$. Thus, $\lim _{\gamma \rightarrow 1^{-}} K(\gamma, 1)=+\infty$.

To prove assertion (b), we recall from Lemma 2.3, $c(\gamma, 1)<a(\gamma, 1)$ with $g^{\prime}(a(\gamma, 1)$; $\gamma, 1)=0$ and $\gamma \in[0,1)$. Also from Corollary 4.2(b) in [4], Proposition 2.1(d) and Figure 2.1(3), it is clear that $c(\gamma, 1)$ and $g(c(0,1) ; 0,1)$ are bounded, for $\gamma$ sufficiently close to $0^{-}$. Now, from

$$
\begin{aligned}
|R(\gamma, 1)-R(0,1)|= & |c(\gamma, 1) g(c(\gamma, 1) ; \gamma, 1)-c(0,1) g(c(0,1) ; 0,1)| \\
\leq & c(\gamma, 1)|g(c(\gamma, 1) ; \gamma, 1)-g(c(0,1) ; 0,1)| \\
& +g(c(0,1) ; 0,1)|c(\gamma, 1)-c(0,1)|
\end{aligned}
$$

and continuous property of $g(c(\gamma, 1) ; \gamma, 1), c(\gamma, 1)$, we obtain $\lim _{\gamma \rightarrow 0^{+}} R(\gamma, 1)=$ $R(0,1)$. The desired assertion of $K(0,1)$ could be obtained from the similar arguments.

It is clear from Corollary 3.5, 3.6 that $\Gamma_{3}$ is also a continuum in the quadrant of $R>0, K>0$ with the endpoint $(R(0,1), K(0.1))$ and the limit point $(\infty, \infty)$. The selected numerical data of $R(0,1), K(0,1)$ for various $\varphi$ are shown in Table 1 .

\subsection{Non-monotone solutions}

Recall that the second roots $c_{2}(\alpha, \beta)$ of $\psi(\xi)=0$, for $(\alpha, \beta) \in D_{4}$, will lead to the non-monotone solutions of (BVP) with positive $R, K$, since $g\left(c_{2}(\alpha, \beta)\right)>0$. In fact, by following the lines of Theorem 3.2, the existence of type (III) solutions can be obtained easily from the next theorem.

Theorem 3.4. (BVP). has a non-monotone solution with suction if and only if $(R, K) \in \Gamma_{4}$. 
As in Sections 3.2, 3.4, the limits of $c_{2}(\gamma, 1)$, and the corresponding $R_{2}(\gamma, 1)$, $K_{2}(\gamma, 1)$ on $\Gamma_{4}$ can be obtained from next two corollaries.

Corollary 3.7. (a). $\lim _{\gamma \rightarrow-1^{+}} c_{2}(\gamma, 1)=+\infty ; \quad$ (b). $\lim _{\gamma \rightarrow 0^{-}} c_{2}(\gamma, 1)=c(0,1)$.

Proof. The assertion (a) is the direct consequence of the fact $c_{2}(\gamma, 1)>c_{1}(\gamma, 1)$ and $\lim _{\gamma \rightarrow-1^{+}} c_{1}(\gamma, 1)=+\infty$. To prove the assertion (b), we again recall that $\lim _{\gamma \rightarrow 0^{-}} a_{2}(\gamma, 1)=a(0,1)$ with $g^{\prime}\left(a_{2}(\gamma, 1) ; \gamma, 1\right)=0$ for $\gamma \in(-1,0)$ from Corollary 5.2(b) in [2]. In fact, $c_{2}(\gamma, 1)<a_{2}(\gamma, 1)$ and $g\left(c_{2}(\gamma, 1) ; \gamma, 1\right)<g\left(a_{2}(\gamma, 1) ; \gamma, 1\right)$. We also have $c(0,1)<a(0,1), g(c(0,1) ; 0,1)<g(a(0,1) ; 0,1)$ with $g^{\prime}(a(0,1) ; 0,1)=0$. This implies that $g^{\prime}(c(0,1)), g^{\prime}\left(c_{2}(\gamma, 1)\right), g^{\prime \prime}\left(c_{2}(\gamma, 1)\right)$ and $g^{\prime \prime}(c(0,1))$ are well-defined. Hence, from

$$
\left|c_{2}(\gamma, 1)-c(0,1)\right|=\frac{1}{\varphi}\left|\frac{g^{\prime}(c(\gamma, 1))}{g^{\prime \prime}\left(c_{2}(\gamma, 1)\right)}-\frac{g^{\prime}(c(0,1))}{g^{\prime \prime}(c(0,1))}\right|
$$

and the continuous dependence, the assertion (b) is obtained.

Now the limiting points $(R(0,1), K(0,1)),(+\infty,+\infty)$ of $\Gamma_{4}$ can be obtained from the next Corollary, where $(R(0,1), K(0,1))$ is defined in Corollary 3.6.

Corollary 3.8. (a). $\lim _{\gamma \rightarrow-1^{+}} R_{2}(\gamma, 1)=+\infty, \lim _{\gamma \rightarrow-1^{+}} K_{2}(\gamma, 1)=+\infty$, for sufficiently small $\varphi$;

(b). $\lim _{\gamma \rightarrow 0^{-}} R_{2}(\gamma, 1)=R(0,1), \lim _{\gamma \rightarrow 0^{-}} K_{2}(\gamma, 1)=K(0,1)$, for some positive $R(0,1), K(0,1)$.

Proof. We omit the verification of the assertion (b), since it is similar to the ones in Corollary 3.6(b). To prove assertion (a), we recall the fact $g\left(a_{2}(\gamma, 1)\right)>1$ for $\gamma \in(-1,0)$, from Corollary 5.3(b) in [4]. It is clear that $\psi(\xi)=\psi(\xi, \varphi)$ and $\psi\left(a_{2}(\gamma, 1), 0\right)=0$. Then, by the implicit function theorem, for sufficiently small $\varphi$, $c_{2}(\gamma, 1):=c_{2}(\gamma, 1 ; \varphi)$ is a continuous function of $\varphi$ with $c_{2}(\gamma, 1 ; 0)=a_{2}(\gamma, 1)$. That is, for sufficiently small $\varphi, g\left(c_{2}(\gamma, 1 ; \varphi)\right)>1$ for $\gamma \in(-1,0)$. Then, the desired limit of $R_{2}(\gamma, 1)$ is obtained.

The assertion $\lim _{\gamma \rightarrow-1^{+}} K_{2}(\gamma, 1)=+\infty$ is easily obtained by the continuous dependence, we get that

$$
\begin{aligned}
g\left(c_{2}(\gamma, 1) ; \gamma, 1\right) & =\int_{0}^{c_{2}(\gamma, 1)} g^{\prime}(\xi) d \xi \leq g^{\prime}\left(d_{2}^{*}(\gamma, 1) ; \gamma, 1\right) c_{2}(\gamma, 1) \\
& =c_{2}(\gamma, 1)\left[\int_{0}^{d_{2}^{*}(\gamma, 1)} g^{\prime \prime}(\xi) d \xi+\gamma\right]
\end{aligned}
$$




$$
\begin{aligned}
& \leq c_{2}(\gamma, 1)\left[d_{2}^{*}(\gamma, 1) g^{\prime \prime}\left(d_{3}^{*}(\gamma, 1) ; \gamma, 1\right)+\gamma\right] \\
& <c_{2}(\gamma, 1)\left[c_{2}^{2}(\gamma, 1)\left(1-\gamma^{2}\right)+\gamma\right] \\
& =c_{2}^{3}(\gamma, 1)\left(1-\gamma^{2}\right)+c_{2}(\gamma, 1) \gamma \\
& \leq c_{2}^{3}(\gamma, 1)\left(1-\gamma^{2}\right)
\end{aligned}
$$

This implies that $1 / R_{2}>1 /\left[c_{2}^{4}(\gamma, 1)\left(1-\gamma^{2}\right)\right]$, and, consequently,

$$
K_{2}=\frac{c_{2}^{4}(\gamma, 1)}{R_{2}}>\frac{c_{2}^{4}(\gamma, 1)}{c_{2}^{4}(\gamma, 1)\left(1-\gamma^{2}\right)}=\frac{1}{\left(1-\gamma^{2}\right)}
$$

when $\gamma$ is sufficiently closed to $-1^{+}$. Thus, $\lim _{\gamma \rightarrow-1^{+}} K_{2}(\gamma, 1)=+\infty$.

Note that for any given positive $\varphi$ the point $(R(0,1), K(0.1))$ on $\Gamma_{3}$ corresponds to a non-negative, non-concave, suctive solution, and therefore, it is a limit point of the continuum $\Gamma_{4}$. Moreover, $(+\infty,+\infty)$ is also a limit point of $\Gamma_{3}$. Furthermore, $\Gamma^{*}=\Gamma_{3} \cup \Gamma_{4}$ forms a continuum in the quadrant of $R>0, K>0$.

\section{Concluding Remarks}

In summary, (BVP) can only possess three different types of solutions, and there exist two continuums $\Gamma_{*}=\Gamma_{1} \cup \Gamma_{2} \cup(0,-3 /(1+3 \varphi)), \Gamma^{*}=\Gamma_{3} \cup \Gamma_{4}$, on which each point corresponds to one solution of certain type. Now, our main result can be easily obtained from the following theorems:

Theorem 4.1. Given any positive $\varphi$, (BVP) possesses at least one nonnegative and concave solution for every real $R$.

It should be pointed out that our numerical simulation has indicated that there exists a turning point $(R(\varphi), K(\varphi))$ on $\Gamma_{4}$, as shown in Figure 3.2, with $R(\varphi)<R(0,1)$ for various positive $\varphi$, and the selected data of $R(\varphi)$ are also shown in Table 1. Therefore, in addition to the type (I) suctive solutions, one can conjecture that (BVP) has at least two suctive solutions for $R \geq R(\varphi)$. However, our mathematical analysis for the existence of type (II), (III) solutions can only be concluded from the following theorem.

Theorem 4.2. Given any sufficiently small $\varphi>0$, (BVP) possesses at least one nonnegative, non-concave suctive solution, and at least one non-monotone suctive solution for $R \geq R(0,1)$, for some positive $R(0,1)$ varying in $\varphi$.

Theorem 4.3. Given any positive $\varphi>0$, (BVP) possesses no solution for $R \leq 0$, $K \geq 0$. 
Also note that the slip coefficient $\varphi$ is in general small. Therefore, our result in Theorem 4.2 is indeed of physical interest. Furthermore, the classification in Section 2 also leads to the following nonexistence result:

Table 1. The selected data of $R(\varphi), R(0,1)$ and $K(0,1)$ for various $\varphi$

\begin{tabular}{|c|c|c|c|}
\hline slip coefficient $\varphi$ & $R(\varphi)$ & $R(0,1)$ & $K(0,1)$ \\
\hline 0.1 & 10.76550192 & 11.79506983 & 8.15546349 \\
\hline 0.5 & 7.29223018 & 9.47002532 & 7.32688514 \\
\hline 1.0 & 5.81827450 & 8.60689265 & 7.12537175 \\
\hline 1.5 & 4.97836919 & 8.25410685 & 7.05187182 \\
\hline 2.0 & 4.38847257 & 7.98255317 & 6.99820150 \\
\hline 3.0 & 3.80720381 & 7.78080318 & 6.95981675 \\
\hline 10.0 & 2.71654423 & 7.44868627 & 6.89916457 \\
\hline
\end{tabular}

It should be addressed that the verification of the precise multiplicity of the solutions of (BVP) is still open, although our numerical simulation has exhibited some monotone phenomena for the continuums.

\section{ACKNOWLEDGMENTS}

The authors are grateful to the reviewer's valuable comments and careful reading.

\section{REFERENCES}

1. A. B. Berman, Laminar flow in channel with porous wall, J. Appl. Phys., 24 (1953), 1232-1235.

2. F. M. Skalak and C. Y. Wang, On the nonunique solutions of laminar flow through a porous tube or channel, SIAMJ. Appl. Math., 34 (1978), 535-544.

3. S. Chellam, M. R. Wiesner and C. Dawson, Slip at a uniformly porous boundary: effect on fluid flow and mass transfer, J. Eng. Mech., 26 (1992), 481-492.

4. T.-W. Hwang and C.-A. Wang, On multiple solutions for Berman's problem, Proc. Roy. Soc. Edinburgh Sect., 121A (1992), 219-230.

5. S. Chellam and Liu Mei, Effect of slip on existence, uniqueness, and behavior of similarity solutions for steady incompressible laminar flow in porous tubes and channels, Phys. Fluid., 18(8) (2006), DOI:10.1063/1.2236302.

Un-Un Kuo and Ching-An Wang

Department of Mathematics

National Chung Cheng University

Ming-hsiung, Chia-Yi 621, Taiwan

E-mail: cawang@mail.stut.edu.tw 